\title{
Learning-Induced Survival of New Neurons Depends on the Cognitive Status of Aged Rats
}

\author{
Elodie Drapeau, ${ }^{\star}$ Marie-Françoise Montaron, ${ }^{\star}$ Sylvie Aguerre, and Djoher Nora Abrous \\ Institut National de la Santé et de la Recherche Médicale U862, Bordeaux Neuroscience Research Center, University of Bordeaux 2, Bordeaux, France
}

Aging is accompanied by an alteration of spatial memory, which has been related to an alteration in hippocampal plasticity. Within the dentate gyrus, new neurons are generated throughout the entire life of an individual. This neurogenesis seems to play a role in hippocampal-mediated learning and learning-induced changes in neurogenesis have been proposed to be involved in memory. However, in aged rats, little is known on the influence of learning on the early development of the adult-born neurons and on the possible involvement of learning-induced changes in neurogenesis in age-related memory deficits. To address this issue, we took advantage of the existence of spontaneous individual differences for performances observed in aged subjects in the water maze. In this task, learning can be divided into two phases, an early phase during which performances quickly improve, and a late phase during which asymptotic levels of performances are reached. We show that the influence of spatial learning on the survival of the newly born cells depends on their birth date and the memory abilities of the aged rats. In aged rats with preserved spatial memory, learning increases the survival of cells generated before learning whereas it decreases survival of cells produced during the early phase of learning. These results highlight the importance of learning-induced changes in adult-born cell survival in memory. Furthermore, they provide new insights on the possible neural mechanisms of aging of cognitive functions and show that an alteration to the steps leading to neurogenesis may be involved in the determination of individual memory abilities.

Key words: neurogenesis; hippocampus; neuroplasticity; aging; spatial learning; memory

\section{Introduction}

Normal aging is generally accompanied by a progressive cognitive decline particularly in memory functions (Grady and Craik, 2000; Small, 2001). In rodents, this decline has been shown in different learning tests especially involving spatial memory like the water maze (Gage et al., 1984; Gallagher et al., 1993). These impairments, similar to those observed after hippocampal lesions (Redish and Touretzky, 1998; Stoelzel et al., 2002), are associated with deteriorations of hippocampal circuitry and plasticity (Geinisman et al., 1986; Fischer et al., 1992; Smith et al., 2000). However, this aging-related alteration of cognitive functions is extremely variable within a population. In the hippocampaldependant version of the water maze, some old animals show a clear impairment in spatial memory, whereas others exhibit capacities similar to those of younger individuals (Gage et al., 1988; Markowska et al., 1989; Rapp and Amaral, 1992; Gallagher et al., 1993). These individual differences analyses have successfully

\footnotetext{
Received June 15, 2006; revised April 17, 2007; accepted April 18, 2007.

This work was supported by Institut National de la Santé et de la Recherche Médicale (INSERM), University of Bordeaux II. We are very grateful to Drs. M. Le Moal, W. Mayo, and P. V. Piazza (INSERM U588) for their comments. The technical help of $D$. Gonzales and M. Kharouby is acknowledged.

${ }^{*}$ E.D. and M.F.M. contributed equally to this work.

Correspondence should be addressed to Dr. Djoher Nora Abrous, Laboratory of Neurogenesis and Pathophysiology, Institut National de la Santé et de la Recherche Médicale U862, Bordeaux Neuroscience Research Center, 146 rue Léo-Saignat, Bordeaux, France. E-mail: Nora.Abrous@bordeaux.inserm.fr.

E. Drapeau's present address: Departments of Pathology, Neurology and Center for Neurobiology and Behavior, Columbia University, P\&S 14-511, 630 West 168th Street, New York, NY 10032.

D0I:10.1523/JNEUROSCI.1031-07.2007

Copyright $\odot 2007$ Society for Neuroscience $\quad 0270-6474 / 07 / 276037-08 \$ 15.00 / 0$
}

linked functional and structural modifications of the hippocampal formation (HF) with age-related disorders.

The dentate gyrus (DG) of the HF is one of the areas in which neurons are generated throughout life (Abrous et al., 2005; Aimone et al., 2006). Neurogenesis has been hypothesized to play a role in hippocampus-mediated learning in young adult rodents. Conditions increasing memory performances also enhance neurogenesis whereas situations reducing neurogenesis induce cognitive impairments (Kempermann et al., 1997; van Praag et al., 1999; Lemaire et al., 2000; Shors et al., 2001). Moreover, spatial learning influences the production and the fate of the newly born cells according to their birth date. Indeed, learning increases the survival of cells born before the learning (Gould et al., 1999; Hairston et al., 2005). However, the survival of cells generated during the early phase of learning (when performances improve rapidly) is decreased by the late phase of learning when performances are stabilized. Furthermore, this late phase increases cell proliferation (Lemaire et al., 2000; Dobrossy et al., 2003).

During aging, little is known about the relationship between neurogenesis and spatial memory. It has been shown that exposure of middle-aged or senescent animals to an enriched environment increases spatial memory abilities and neurogenesis (Kempermann et al., 1998, 2002). Furthermore, neurogenesis is positively correlated to performances in the water maze (Drapeau et al., 2003). Finally, lowering corticosterone levels in midlife increases hippocampal neurogenesis and spatial memory performances (Montaron et al., 2006). However, it remains unknown whether spatial learning in aged animals influences birth and/or 
survival of adult-born cells and whether these dynamic changes depend on memory abilities.

To address this issue, we analyzed the influence of spatial learning on the production, survival, and fate of cells, depending on their birth date. Cell genesis was studied by injecting bromodeoxyuridine to date the birth of cells produced before, during, or after the training. We showed that, in aged rats with preserved performances, learning increases the survival of cells generated before learning, but decreases the survival of cells produced during the early phase. These results provide new insights as to the possible neural mechanisms of aging of spatial memory and show that alterations of the chain of events leading to neurogenesis may be involved in spatial hippocampal-dependant memory abilities.

\section{Materials and Methods}

Animals. One hundred and thirty male Sprague Dawley rats (Iffa Credo, Lyon, France) between 11 and 12 months of age were purchased and maintained undisturbed in our facilities under a constant $12 \mathrm{~h} \mathrm{light/dark}$ cycle (light on from 8 A.M. to 8 P.M.) with ad libitum access to food and water. Temperature $\left(22^{\circ} \mathrm{C}\right)$ and humidity $(60 \%)$ were kept constant. Four weeks before the start of the experiments, animals were housed individually and handled. Animals with bad general health status or tumor were excluded. All experiments were conducted in strict compliance with the recommendations of the European Union (86/609/EEC) and the French National Committee (87/848).

Water-maze training. At 20 months of age, rats $(n=93)$ were trained in a Morris water maze according to a previously described method (Drapeau et al., 2003). Briefly, the pool (180 cm diameter, $60 \mathrm{~cm}$ high) was filled with water $\left(21^{\circ} \mathrm{C}\right)$ made opaque by addition of milk powder. An escape platform was hidden $2 \mathrm{~cm}$ below the surface of the water in a fixed location in one of four quadrants, halfway between the wall and the middle of the pool. Before the start of training, animals were habituated to the pool without platform $1 \mathrm{~min}$ per day for $3 \mathrm{~d}$. During training, animals were required to locate the submerged platform using distal extramaze cues. They were all tested for four trials per day $(90 \mathrm{~s}$ with an intertrial interval of $30 \mathrm{~s}$ and beginning from three different start points that varied randomly each day). If an animal did not find the platform, it was set on it at the end of the trial. The time to reach the platform (latency in seconds) and the length of the swim path (distance in meters) were measured with a computerized tracking system (Videotrack; Viewpoint, Lyon, France). Daily results were analyzed to rank animals according to their behavioral score. In the first experiment, all animals were trained for $8 \mathrm{~d}$ and were not allowed to reach asymptotic levels of performances. Asymptotic performance was defined when performances were stable for at least for 3 training days. Animals from experiments 2-4 were trained in the water maze until the aged unimpaired rats (with behavioral scores below the median of the group) reached at least $3 \mathrm{~d}$ of stable performances (with no statistical significant difference between days). Aged impaired rats (with behavioral scores above the median of the group) did not maintain a stable performance. Untrained (UnT) animals were left in the animal house and were not exposed to the water maze.

5-Bromo-2'-deoxyuridine injections. 5-Bromo-2'-deoxyuridine (BrdU; Sigma, St. Louis, MO), a thymidine analog incorporated into genetic material during the $S$ phase of the cell cycle, was used to label dividing cells. Animals received one daily intraperitoneal injection of 50 $\mathrm{mg} / \mathrm{kg}$ BrdU during $5 \mathrm{~d}$ according to a previously described method (Drapeau et al., 2003). Trained animals were injected 1 week before the behavioral task (first experiment), during the first $5 \mathrm{~d}$ of the training (second experiment), during the last $5 \mathrm{~d}$ of the training (third experiment), or $9 \mathrm{~d}$ after the completion of the task (fourth experiment). Rats in the second and third experiments were injected 30 min before the session and were replaced in their home cage until the beginning of the training. Untrained animals received BrdU injections in the same daytime period as the trained animals.

Immunostaining. Animals were deeply anesthetized with an overdose of sodium pentobarbital and perfused transcardially with $300 \mathrm{ml}$ of PBS,
pH 7.3, containing heparin $(5 \times 104 \mathrm{IU} / \mathrm{ml})$, followed by $600 \mathrm{ml}$ of $4 \%$ paraformaldehyde in $0.1 \mathrm{~m}$ phosphate buffer, $\mathrm{pH}$ 7.3. After a $24 \mathrm{~h}$ postfixation period, $50 \mu \mathrm{m}$ frontal sections were cut on a vibratome and collected in PBS, pH $7.4(0.1 \mathrm{~m})$. For BrdU and Ki67 labeling, one in 10 free-floating sections was processed according to a standard immunohistochemical procedure (Drapeau et al., 2003; Montaron et al., 2006) using a mouse anti-BrdU monoclonal antibody or a mouse anti-Ki-67antibody (both at 1:100; Dako, Glostrup, Denmark) visualized with the biotinstreptavidin technique (ABC kit; Dako) by using 3,3-diaminobenzidine as chromogen.

The neuronal phenotype of the newly born cell was examined by immunofluorescence double-labeling on adjacent sections by using rat monoclonal anti-BrdU antibody (1:500; Accurate Scientific, Westbury, $\mathrm{NY}$ ), revealed with a CY3 anti-rat antibody (1:1000; Jackson ImmunoResearch, West Grove, PA), combined with a goat polyclonal antidoublecortin (DCX) antibody (1:200; Santa Cruz Biotechnology, Santa Cruz, CA) or with a mouse monoclonal anti-neuronal-specific nuclear protein (NeuN) antibody (1:1000; Millipore Euromedex, Souffelweyersheim, France) revealed, respectively, with an Alexa 488 anti-goat antibody or an Alexa 488 anti-mouse antibody (1:1000; Jackson ImmunoResearch).

Labeling quantification. The number of BrdU-immunoreactive (IR) cells in the DG was estimated by using a modified version of the optical fractionator method on a systematic random sampling of every 10 th 50 $\mu \mathrm{m}$ section along the rostrocaudal axis of the HF (Drapeau et al., 2003). On each section, all BrdU-IR cells were counted with a $\times 100$ microscope objective in the granule and subgranular layers of the left and right DG and in the hilus, excluding those in the outermost focal plane. Resulting numbers were tallied and multiplied by the inverse of the sectionsampling fraction $(1 / \mathrm{ssf}=10)$. Adjacent sections were counterstained and the surface of the granule cell layer (GCL) was measured using a StereoInvestigator System (MicroBrightfield, Williston, VT) and reference volume estimated using the Cavalieri method: Vref $=T \times \Sigma A \times$ (1/ssf), where $T$ is the mean thickness of the vibratome section $(50 \mu \mathrm{m})$ and $A$ is the area of the granule and subgranular cell layers.

The percentage of BrdU-labeled cells that expressed the neuronal markers DCX or NeuN was determined throughout the dentate gyrus by using a confocal microscope with $\mathrm{HeNe}$ and argon lasers (DMR SP2 AOBS, Leica Nussloch, Germany; or LSM 510 META, Zeiss, Oberkochen, Germany). All BrdU-labeled cells were examined except those located in the middle part of the section, where penetration of DCX or NeuN antibodies was not reliable. Sections were optically sliced in the $z$ plane by using a $1 \mu \mathrm{m}$ interval, and cells were rotated in orthogonal planes to verify double labeling.

General procedures. For each experiment, two experimental groups were used: one trained group submitted to the spatial learning, and one control or untrained group made of animals of the same age that were injected with BrdU and perfused at the same time as the trained animals, but which were never exposed to the water maze. In each trained group, all animals were submitted to the same amount of training.

First experiment: effect of spatial learning on the survival of newly born cells. We examined the influence of spatial learning in a water maze on the survival of previous newly born cells in aged rats. Twenty-month-old rats (trained group; $n=27$ ) received BrdU injections for $5 \mathrm{~d}$. They were left undisturbed for $8 \mathrm{~d}$ and then all of the animals of the trained group were trained during $8 \mathrm{~d}$ in the water maze. Animals were perfused after the last day of training. Seven animals were used for the control untrained group and were left undisturbed during the whole procedure.

Second experiment: effect of spatial learning on the number of cells produced during the early phase of learning. We studied the influence of spatial learning in the water maze on the number of newly born cells produced during the early phase of the learning. Twenty-one-month-old rats (trained group; $n=22$ ) were injected with BrdU during the first $5 \mathrm{~d}$ of a 12-d-long training period. Animals were perfused after the last day of training. Nine animals were used for the control untrained group.

Third experiment: effect of spatial learning on the number of cells produced during the late phase of learning. We assessed the influence of spatial learning in the water maze on the number of newly born cells produced during the late phase of the learning. Twenty-one-month-old rats 
(trained group; $n=26$ ) were injected with BrdU during the last $5 \mathrm{~d}$ of an 11-d-long training period. Animals were killed after the last day of training. Fourteen animals were used for the control untrained group.

Fourth experiment: effect of spatial learning on the number of cells produced after the training. To determine whether the production of new cells is influenced after learning, 21-month-old rats were injected with BrdU (for $5 \mathrm{~d}$ ) $9 \mathrm{~d}$ after a 10 -d-long training period (trained group; $n=$ 18). Animals were killed the day after the last BrdU injection. Seven animals were used for the control untrained group.

Statistical analysis. Data were analyzed by an ANOVA followed by a post hoc comparison using the Newman-Keuls test when appropriate. Relationships between learning performances and anatomical parameters were evaluated using the Pearson's correlation test. All data are reported as mean $\pm \mathrm{SEM}$.

\section{Results}

Learning increases the survival of cells whose birth predates the beginning of training in aged rats with preserved spatial memory (first experiment)

In the water-maze task, animals are required to locate a hidden platform using the spatial cues available in the testing room. Learning of this task is reflected by a progressive decrease in the latency (or the distance) to find the escape platform. The evolution of performance is characterized by two consecutive phases. During the early phase, there is a fast and large (>80\%) improvement in performance. During the late phase, there is a smaller increase in performance $(<20 \%)$ and a stable baseline is reached.

In young adult rats, the early phase of learning has been shown to increase the number of new cells that are produced 1 week before exposure to the water maze (Gould et al., 1999; Hairston et al., 2005). Here, rats were injected with BrdU and all rats of the training group were exposed to the water maze (four daily trials for 8 d) $8 \mathrm{~d}$ after the last BrdU injection (first experiment) (Fig. $1 a)$ and were killed before the stabilization of the performances (after eight training days). It has been well established that there are important individual differences in spatial memory abilities (Markowska et al., 1989; Rapp and Amaral, 1992; Gallagher et al., 1993). Indeed, in the water maze, some old individuals show a clear impairment in spatial reference memory, whereas others are not impaired and exhibit cognitive capacities similar to those of younger individuals. The analysis of such individual differences is considered a valuable strategy for the study of the neurobiological substrates of cognitive aging. To take interindividual differences in learning abilities of aged rats into account, the number of BrdU-IR cells was correlated with individual performances. A significant negative correlation was found between the mean (i.e., the mean of the four daily trials over the whole duration of the training) latency and distance to reach the hidden platform and the number of BrdU-IR cells in the GCL (Figs. 1b,c, 2a) (latency, $r=-0.535, p=0.004$; distance, $r=-0.523, p=0.004)$; the highest numbers of BrdU-IR cells were found in rats having the best performances in the water maze [i.e., the shortest latencies (or distances) to reach the platform]. This indicates that learning and not training increased the survival of the cells generated before exposure to the task. In contrast, there was no correlation between the behavioral performances and the reference volume (latency, $r=-0.118, p=0.558$; distance, $r=-0.207, p=0.301$ ).

To further characterize the relationships between neurogenesis and learning, two subgroups of animals were selected on the basis of their behavioral performances (either below or above the median of the group) (Piazza et al., 1989; Lemaire et al., 1999). To this end, on the basis of the mean latency (i.e., mean of the four daily trials over the whole duration of the training), one group of animals was classified as aged unimpaired (AU) $(n=14)$ and the

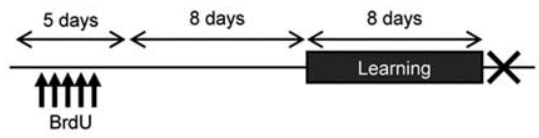

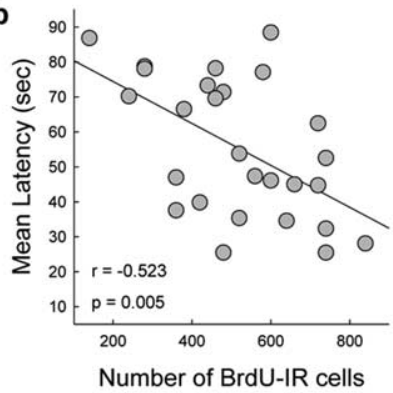

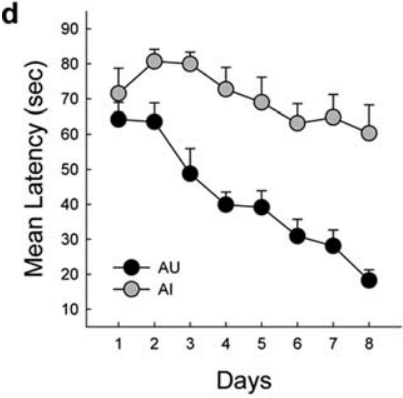

c

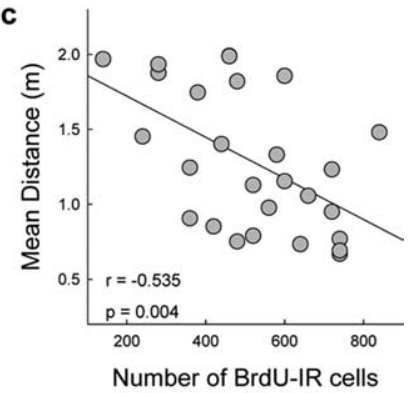

e

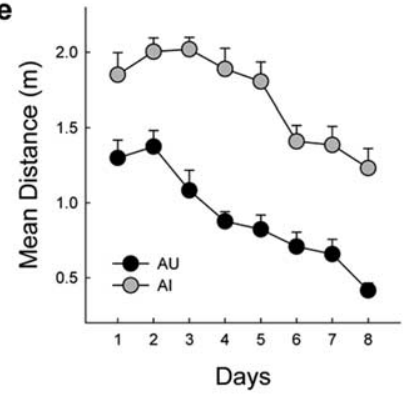

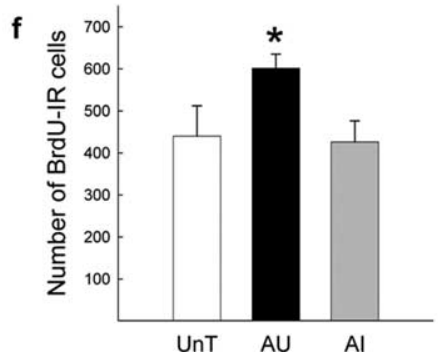

Figure 1. Spatial learning increases the survival of neurons whose birth predates the beginning of training in aged rats with a preserved spatial memory. $\boldsymbol{a}$, Experimental protocol of the first experiment, the arrows indicate the BrdU injections, the cross indicates the day the animals were killed. $\boldsymbol{b}, \boldsymbol{c}$, Correlation between individual performances in the water maze measured by the mean latency $(\boldsymbol{b})$ or distance $(\boldsymbol{c})$ to reach the hidden platform and the total number of BrdU-IR cells in the GCL of the dentate gyrus of aged rats. $\boldsymbol{d}, \boldsymbol{e}$, Spatial learning as shown by the evolution of the mean latency $(\boldsymbol{d})$ or distance $(\boldsymbol{e})$ to find the hidden platform for $\mathrm{AU}$ and $\mathrm{Al}$ rats. $\boldsymbol{f}$, Total number of BrdU-IR cells in the GCL of UnT, AU, and Al rats. ${ }^{*} p<0.05$ compared with the other groups.

other group was classified as aged impaired (AI) $(n=13)$. As expected, the evolution of performance of the AU and AI groups was different: in the AU group, there was a dramatic improvement in performance, which was not the case for the AI group (Fig. 1d,e). These differences in learning were not caused by any motor-performance alterations because the swim speeds did not differ between groups (AI, $25.72 \pm 2.42 \mathrm{~cm} / \mathrm{s}$; AU, $22.38 \pm 1.04$ $\left.\mathrm{cm} / \mathrm{s} ; t_{(25)}=-1.301 ; p=0.205\right)$.

Trained animals were compared with UnT animals for their BrdU-IR cell number. The number of BrdU-IR cells in the GCL of AU rats was significantly increased compared with UnT and AI animals (Fig. $1 f)\left(F_{(2,31)}=4.499 ; p=0.019 ; \mathrm{UnT}=\mathrm{AI}<\mathrm{AU}\right.$ at $p<0.05)$. Thus, animals with the best performances exhibited the highest number of BrdU-IR cells whereas rats with the worst performances exhibited a number of BrdU-IR cells similar to that measured in UnT rats. These results were not caused by a difference in the reference volume (UnT group, $4.439 \pm 0.215 \mathrm{~mm}^{3}$; 
AI group, $4.367 \pm 0.201 \mathrm{~mm}^{3}$; AU group, $4.683 \pm 0.192 \mathrm{~mm}^{3} ; F_{(2,31)}=0.743 ; p=$ $0.484)$.

We then determined the phenotype of the newly born cells that have survived after the training. For this purpose, we used an immature neuronal marker, DCX, and a mature neuronal marker, NeuN. We found that few BrdU-labeled cells expressed DCX (UnT group, $4.42 \pm 3.14 \%$; AI group, $3.59 \pm 1.42 \%$; AU group, $\left.4.50 \pm 3.44 \% ; F_{(2,14)}=0.040 ; p=0.961\right)$. In contrast, about one-half of the population of the newly generated cells expressed NeuN, indicating differentiation to a neuronal phenotype (Fig. 2d) (UnT group, $40.25 \pm 11.61 \%$; AI group, $53.22 \pm$ 14.34\%; AU group, $36.62 \pm 5.48 \% ; F_{(2,17)}$ $=0.712 ; p=0.505$ ).

At this time point, the number of proliferating cells assessed by Ki67 (Endl et al., 2001) immunolabeling in the GCL of AU rats (Fig. 2b) (183.57 \pm 2.73 ) was not different from that measured in AI group $(203.85 \pm 31.65)$ and in UnT group $\left(224.29 \pm 41.91 ; F_{(2,31)}=0.377 ; p=\right.$ $0.689)$. The number of Ki67-IR cells was not related to the learning abilities because no correlation between the behavioral performances and the number of Ki67-IR cells was observed (latency, $r=0.085, p=0.675$; distance, $r=$ $0.236, p=0.236)$.

All together, these data show that, in aged rats with preserved spatial memory, the early phase of learning, during which performances dramatically improve, increased the survival of neurons whose birth date predated the learning. In contrast, cell proliferation was not changed by learning when measured at the end of training.

\section{Learning decreases the number of BrdU-IR cells produced during the early phase of spatial learning in aged rats with preserved spatial memory (second experiment)}

We have shown in young adult rats that the late phase of learning, characterized by a lack of additional improvement of the performances, decreased the number of cells produced during the early phase of learning (Döbrössy et al., 2003). To study the influence of spatial learning on the number of BrdU-IR cells produced during this early phase of learning, aged animals were injected with BrdU during the first $5 \mathrm{~d}$ of training (Fig. $3 a$, second experiment). All animals were killed when the AU group exhibited $3 \mathrm{~d}$ of stable performances. A significant negative correlation was found between the mean (i.e., mean of the four daily trials over the whole duration of the training) latency and mean distance to reach the hidden platform, and the number of BrdU-IR cells in the GCL (Figs. $2 c, 3 b, c$ ) (latency, $r=0.496, p=0.019$; distance, $r=0.437, p=0.042$ ). This indicates that learning and not training decreased the number of the cells generated during the early phase of the task. In contrast, there was no correlation between the behavioral scores and the number of BrdU-IR cells in the hilus (latency, $r=0.025, p=0.913$; distance, $r=0.084, p=$ 0.710 ) or the reference volume (latency, $r=-0.017, p=0.941$; distance, $r=-0.049, p=0.827)$.

Then, as described previously, trained animals were split into
AI $(n=11)$ and AU $(n=11)$ subgroups (Fig. $3 d, e)$. Although both groups of aged rats acquire the task and are able to reach an asymptotic level of performance, animals from the AU group have a faster improvement of their performance in the water maze than AI animals, and the mean of latencies or distance on the last $3 \mathrm{~d}$ of training (when animal performances are stabilized) is lower for aged unimpaired animals than for aged impaired rats. These differences in learning were not related to differences in swim speed (AI, $25.40 \pm 0.77 \mathrm{~cm} / \mathrm{s} ; \mathrm{AU}, 26.72 \pm 0.80 \mathrm{~cm} / \mathrm{s} ; t_{(20)}$ $=-1.198 ; p=0.245)$. Trained animals were compared with UnT animals for their BrdU-IR cell number. As shown in Figure 3f, AU rats had the lowest number of newly born cells when compared with the AI and UnT groups $\left(F_{(2,28)}=8.028 ; p=0.001\right.$; with $\mathrm{UnT}=\mathrm{AI}<\mathrm{AU}$, at least $p<0.05)$. Thus, animals with the best performances exhibited the lowest number of BrdU-IR cells. In contrast, in rats that did not learn to quickly locate the platform, the number of BrdU-IR cells was similar to that measured in UnT rats and, thus, was unaffected by water-maze training. These differences in BrdU-IR cell number were not caused by a difference in the reference volume (UnT group, $3.963 \pm 0.133 \mathrm{~mm}^{3}$; AI group, $3.619 \pm 0.052 \mathrm{~mm}^{3}$; AU group, $3.715 \pm 0.188 \mathrm{~mm}^{3}$; $\left.F_{(2,28)}=1.568 ; p=0.226\right)$. They were specific of the GCL as BrdU-IR cell number in the hilus was similar among groups (UnT group, $2203.33 \pm 159.56$ BrdU-IR cells; AI group, $1925.45 \pm 227.23$ BrdU-IR cells; AU group, $1843.64 \pm 207.09$ BrdU-IR cells; $\left.F_{(2,28)}=0.785 ; p=0.466\right)$.

We then determined the phenotype of the newly born cells using DCX or NeuN. We found that few BrdU-labeled cells expressed DCX (UnT group, $3.86 \pm 0.82 \%$; AI group, $4.09 \pm$ $1.96 \%$; AU group, $\left.4.57 \pm 1.80 \% ; F_{(2,28)}=0.045 ; p=0.956\right)$. In contrast, $\sim 40 \%$ of the population of newly generated cells expressed NeuN (Fig. 2d) (UnT group, $37.98 \pm 14.72 \%$; AI group, 
a

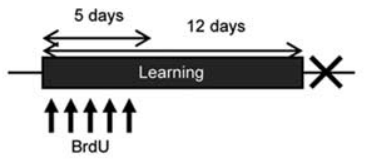

b

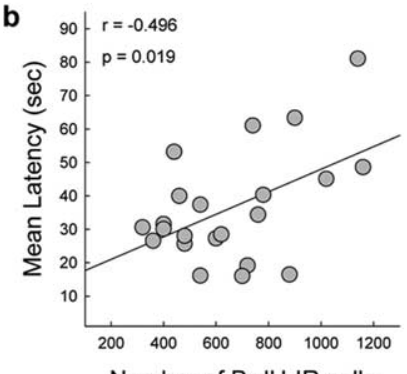

Number of BrdU-IR cells
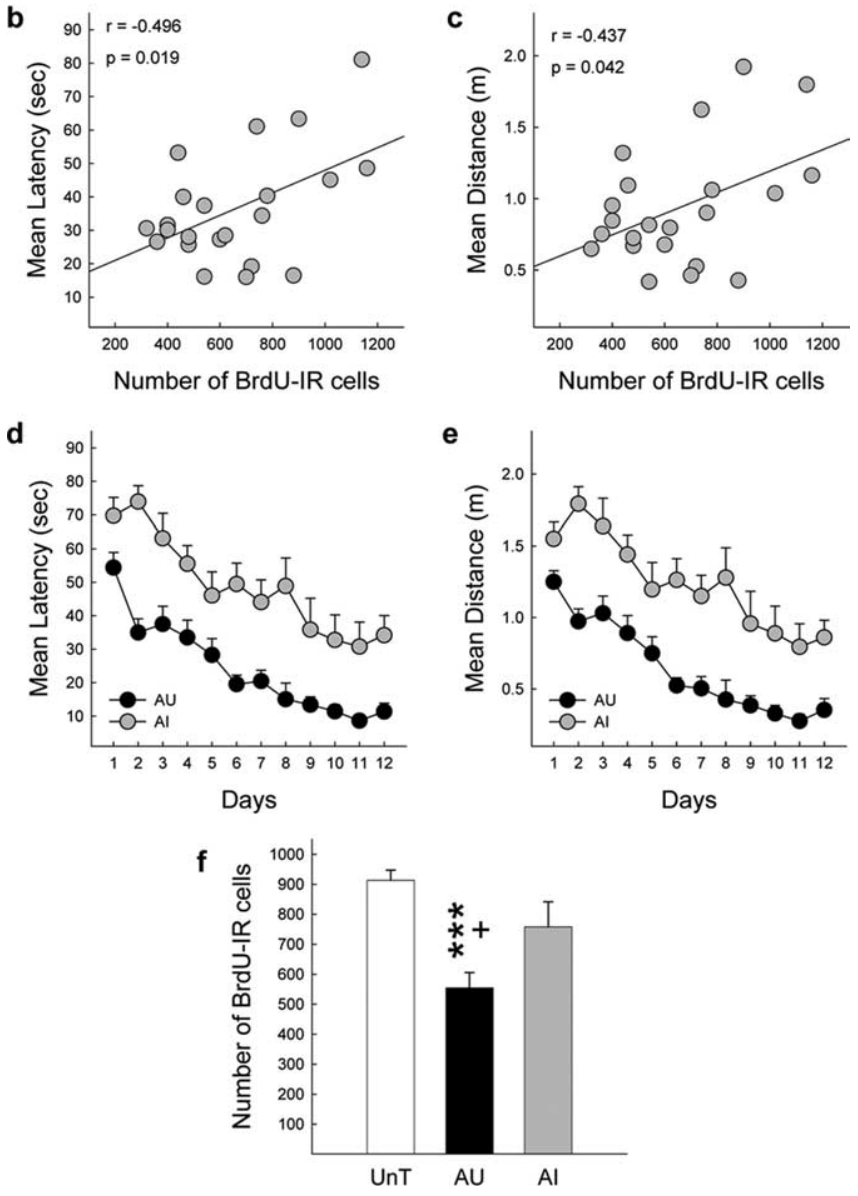

Figure 3. Spatial learning decreases the number of neurons produced during the early phase of learning in aged rats with a preserved spatial memory. $\boldsymbol{a}$, Experimental protocol of the second experiment, the arrows indicate the BrdU injections, the cross indicates the day the animals were killed. $\boldsymbol{b}, \boldsymbol{c}$, Correlation between individual performances in the water maze measured by the mean latency $(\boldsymbol{b})$ or distance $(\boldsymbol{c})$ to reach the hidden platform and the total number of BrdU-IR cells in the GCL of the dentate gyrus of aged rats. $\boldsymbol{d}$, $\boldsymbol{e}$, Spatial learning as shown by the evolution of the mean latency $(\boldsymbol{d})$ or distance $(\boldsymbol{e})$ to find the hidden platform for ages $\mathrm{AU}$ and $\mathrm{AI}$ rats. $\boldsymbol{f}$, Total number of BrdU-IR cells in the $\mathrm{GCL}$ of UnT, AU, and Al rats. ${ }^{+} p<0.05$ compared with Al group; ${ }^{* *} p<0.001$ compared with UnT group.

$36.06 \pm 7.03 \%$; AU group, $37.05 \pm 5.40 \% ; F_{(2,28)}=0.011 ; p=$ $0.989)$.

In conclusion, the number of adult-born cells produced during the early phase of learning was influenced by the water-maze training and by spatial memory abilities of the aged rats.

Learning does not modify the number of BrdU-IR cells produced during the late phase (third experiment)

We have demonstrated that, in young rats, the late phase of learning increased the number of new cells produced contingently with this phase (Lemaire et al., 2000; Döbrössy et al., 2003). Thus, here we studied the influence of a spatial learning on the number of BrdU-IR cells produced during the late phase of training by injecting aged rats with BrdU during the last $5 \mathrm{~d}$ of the learning when a stable baseline of performances was reached (third experiment) (Fig. 4a). Despite important interindividual variations in

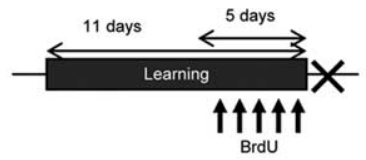

b

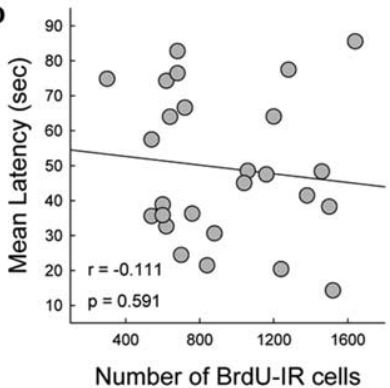

d

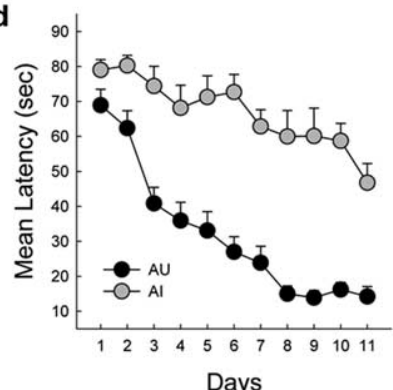

c

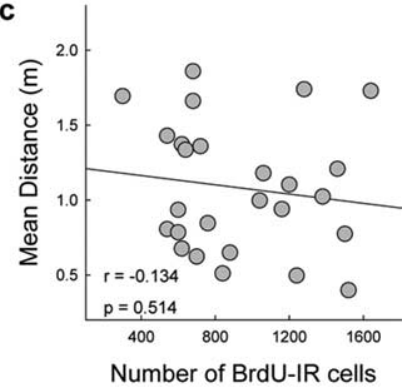

e

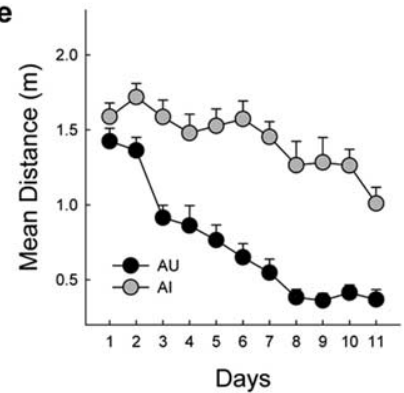

f

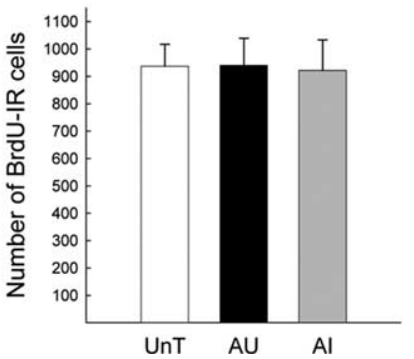

Figure 4. Spatial learning does not modify the number of new neurons produced during the late phase of learning. $\boldsymbol{a}$, Experimental protocol of the third experiment, the arrows indicate the BrdU injections, the cross indicates the day the animals were killed. $\boldsymbol{b}, \boldsymbol{c}$, Correlation between individual performances in the water maze measured by the mean latency $(\boldsymbol{b})$ or distance $(\boldsymbol{c})$ to reach the hidden platform and the total number of BrdU-IR cells in the $\mathrm{GCL}$ of the dentate gyrus of aged rats. $\boldsymbol{d}, \boldsymbol{e}$, Spatial learning as shown by the evolution of the mean latency $(\boldsymbol{d})$ or distance (e) to find the hidden platform for $\mathrm{AU}$ and $\mathrm{Al}$ rats. $\boldsymbol{f}$, Total number of BrdU-IR cells in the $\mathrm{GCL}$ of UnT, AU, and Al rats.

the trained group, no correlation was found between the individual learning performances measured by the mean (i.e., the mean of the four daily trials over the whole duration of the training) latency or distance to reach the platform and the number of newly born cells in the GCL (Fig. 4b,c) (latency, $r=-0.111, p=0.591$; distance, $r=-0.134, p=0.514$ ), in the hilus (latency, $r=0.022$, $p=0.916$; distance, $r=-0.003, p=0.987)$, or the reference volume (latency, $r=-0.002, p=0.991$; distance, $r=-0.013$, $p=0.951)$.

The separation of trained group into AI and AU subgroups (Fig. $4 d, e)$ ( $n=13$ for each group) showed that AU rats mastered the task, reaching an asymptotic level of performances for the 3 last days of training, which was not the case of the AI rats. These differences in learning were not attributable to any motorperformance alterations because the swim speeds did not differ between groups (AI, $21.58 \pm 0.66 \mathrm{~cm} / \mathrm{s} ; \mathrm{AU}, 23.25 \pm 0.58 \mathrm{~cm} / \mathrm{s}$; $\left.t_{(24)}=1.896 ; p=0.583\right)$. There was no difference between the 
different groups regarding the number of BrdU-IR cells in the GCL (Fig. 4f) $\left(F_{2,37}=0.010 ; p=0.990\right)$. Finally, we then determined the phenotype of the newly born cells using DCX and found that $10 \%$ of the BrdU-labeled cells expressed DCX (UnT group, $10.96 \pm 4.09 \%$; AI group, $10.85 \pm 2.04 \%$; AU group, $\left.11.43 \pm 2.42 \% ; F_{(2,13)}=0.011 ; p=0.986\right)$.

These data show that in the aged brain, the late phase of learning did not increase the proliferation of the cells produced contiguously with this phase.

\section{Learning modifies the number of BrdU-IR cells produced after the late phase of spatial learning in aged rats with deficits in spatial memory (fourth experiment)}

These results indicate that the number of BrdU-IR cells produced during the late phase of learning was not influenced by the watermaze training and by the cognitive status of the aged rats. However, given that neurogenesis is retarded by aging (Heine et al., 2004), we hypothesized that learning-induced increase in cell proliferation may also be delayed in the old brain. To address this issue, another batch of trained animals (fourth experiment) was injected with BrdU 9-14 d after the completion of the 10-d-long training period (Fig. $5 a$ ). In these conditions, unexpectedly the behavioral scores were positively correlated with the number of BrdU-IR cells in the DG (Fig. 5b,c) (latency, $r=0.475, p=0.046$; distance, $r=0.508, p=0.031)$. After ranking trained animals on the basis of their mean latency to reach the hidden platform during the whole training (Fig. $5 d, e)(n=9$ for each group), we found that AI rats had the highest number of newly born cells when compared with the AU and UnT groups (Fig. $5 f)\left(F_{(2,22)}=\right.$ 5.515; $p=0.011$; with $\mathrm{UnT}=\mathrm{AU}<\mathrm{AI}$ at least $p<0.05)$. These differences could be attributable to the fact that AU animals $(24.75 \pm 0.72 \mathrm{~cm} / \mathrm{s})$ were more active in the water maze compared with the AI animals $\left(20.53 \pm 1.63 \mathrm{~cm} / \mathrm{s} ; t_{(16)}=-2.359 ; p=\right.$ 0.031).

All together, data from the third and fourth experiments show that, in the aged brain, the late phase of learning did not increase cell proliferation of the cells produced contiguously with this phase. Yet, the water-maze training induced a late bounce of cell proliferation in animals that were unable to master the task during the training.

\section{Discussion}

By analyzing the interindividual differences that are spontaneously observed within a population of aged rats, our data show for the first time that the survival of newly born cells is decreased or increased after learning and depending on the birth date and memory abilities of aged rats. In rats showing the best behavioral performances, learning increased the survival of cells generated before training whereas it decreased the survival of cells produced during the early phase of training. However, learning did not modify neuronal determination and cell proliferation.

The production of new neurons in the DG results from cell proliferation and survival of the newly born cells. Here, we showed that, independently of the learning abilities of the aged rats, learning did not increase the proliferation of cells generated contingently during the early (first experiment) or the late phase (third experiment) of learning. This indicates that BrdU availability and metabolism is independent of spatial memory abilities, thereby confirming previous observations (Drapeau et al., 2003). However, these results are in contradiction to those obtained in young rats showing that the late phase of learning increases cell proliferation that occurs during this phase (Lemaire et al., 2000; Döbrössy et al., 2003). Furthermore, the lack of
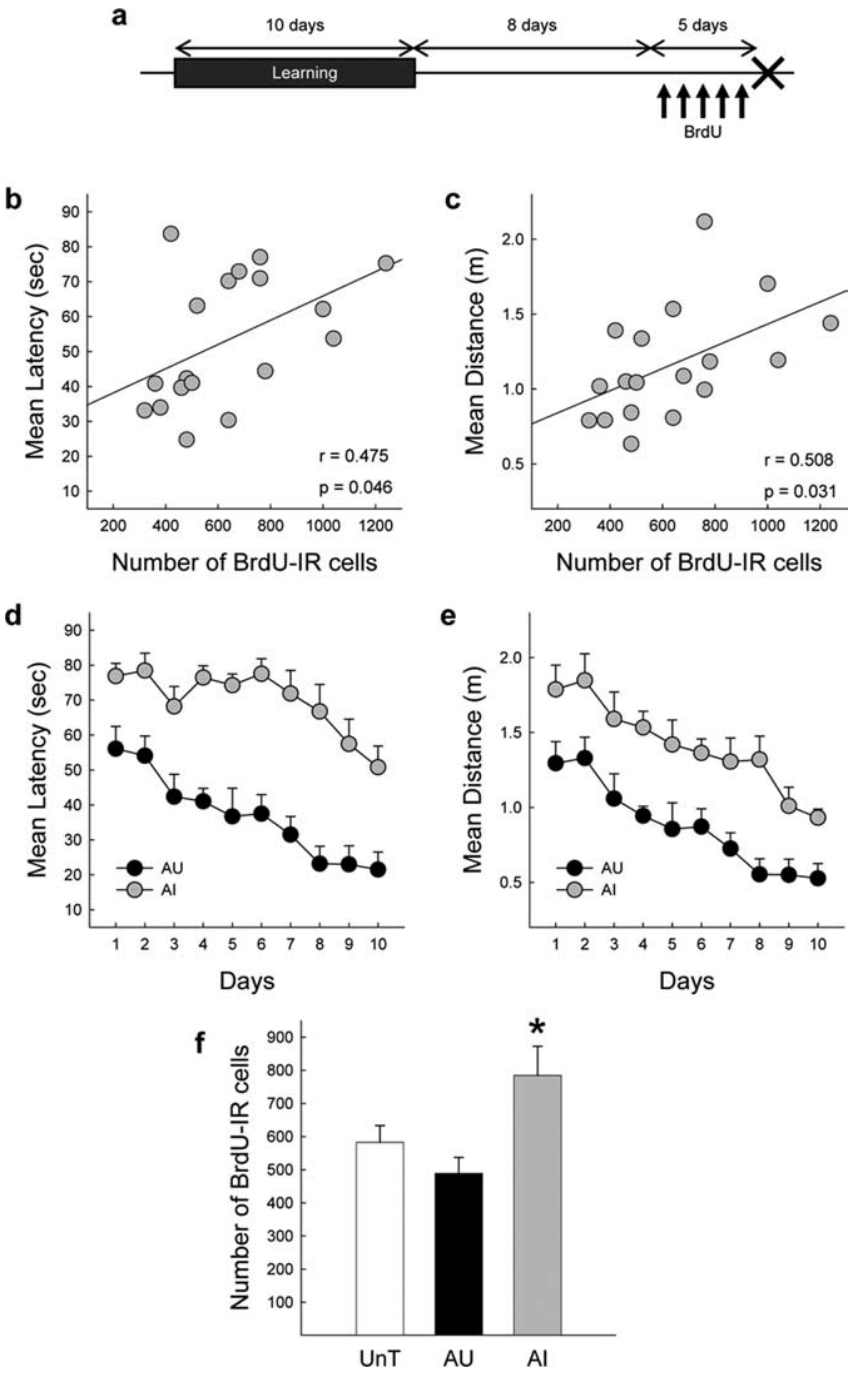

Figure 5. Spatial learning modifies the number of cells produced between 9 and $14 \mathrm{~d}$ after the training. $\boldsymbol{a}$, Experimental protocol of the fourth experiment. The arrows indicate the BrdU injections, the cross indicates the day the animals were killed. $\boldsymbol{b}, \boldsymbol{c}$, Correlation between individual performances in the water maze measured by the mean latency $(\boldsymbol{b})$ or distance $(\boldsymbol{c})$ to reach the hidden platform and the total number of BrdU-IR cells in the $\mathrm{GCL}$ of the dentate gyrus of aged rats. $\boldsymbol{d}, \boldsymbol{e}$, Spatial learning as shown by the evolution of the mean latency $(\boldsymbol{d})$ or distance (e) to find the hidden platform for aged $A U$ and $A I$ rats. $f$, Total number of BrdU-IR cells in the GCL of UnT, AU, and Al rats. * $p<0.05$ compared with AU and UnT groups.

learning-induced cell proliferation in rats with preserved spatial memory indicated that this process did not sustain ongoing learning. This is not surprising because in young rats learninginduced cell proliferation was not correlated to learning performances (Döbrössy et al., 2003). Given that during aging, the different steps leading to hippocampal neurogenesis are retarded (Lichtenwalner et al., 2001; Nacher et al., 2003; Heine et al., 2004), we hypothesized that the lack of learning-induced increase in cell proliferation might be related to a delayed capacity of the senescent brain to respond to learning-triggered signals. However, this did not seem to be the case because 9-14 $\mathrm{d}$ after the completion of the task, cell genesis was not modified in rats with preserved spatial memory, indicating that this process did not sustain ongoing learning. Unexpectedly, cell proliferation was increased in AI rats that exhibited, in this experiment, a higher swimming activity compared with AU rats. Because physical exercise increases cell proliferation (van Praag et al., 1999), this may explain this late bounce of proliferation. 
The influence of learning was examined on the survival of cells generated before or during learning. We observed an opposite effect on the fate of cells depending on their birth date and the cognitive status of the rats. The number of cells generated 9-13 d before exposure to the task was higher in AU rats when compared with the control group and the AI rats. It is likely that these changes in BrdU-labeled cell number resulted from an enhancement of cell survival, as opposed to a further division of the BrdU-labeled cells, because cell proliferation assessed by the number of Ki67-labeled cells was not modified in the same animals. We were not able to determine to what extent a reduction of apoptosis was responsible for the increased survival of BrdUlabeled cells, most probably because of the limits of our detection methods or difference in the kinetics between the two phenomena in the aged brain. Nevertheless, the surviving cells differentiated into neurons. Thus, this survival-promoting effect of learning concerned new-born neurons that were 2 to 3 weeks old during the training. The fact that, in the aged brain, memory abilities were positively correlated to learning-induced increase in the survival of the cells whose birth predated the beginning of learning highlights the importance of this phenomena in the ongoing behavior for the establishment of a spatial map. Consistent with the present results, it has been shown that, in young rats, $4 \mathrm{~d}$ of training in the water maze also increased the survival of cells labeled 1 week before training (Gould et al., 1999; Hairston et al., 2005). Associative learning also increases the survival of newly born neurons generated at least $7 \mathrm{~d}$ before the learning (Gould et al., 1999; Olariu et al., 2005). The fact that few studies did not replicate this finding in young rats has been discussed previously (Abrous and Wojtowicz, 2007).

In contrast, the late phase of learning decreased the number of newly born BrdU-IR cells that were produced during the early phase of learning. The extent of this decrease was correlated with behavioral performances: it was present in AU rats and absent in AI rats. It could be hypothesized that this decrease in the number of BrdU-IR cells may be caused by a difference in BrdU metabolism and/or the stress generated by the water maze, and/or to a dilution of BrdU-labeling as a consequence of further divisions of labeled cells during the late phase of learning, which may render the staining undetectable. However, these possibilities seem unlikely. First, given that the number BrdU-labeled cells present in the hilus was similar in AI and AU animals, a possible difference in BrdU availability is ruled out (see above) (Drapeau et al., 2003). Second, the number of newly born cells in AI rats that had the same training experience as the AU group did not differ from the ones of untrained control group. Thus, a stress component cannot explain the observed effects. Third, neither the early phase nor the late phase of learning modified the number of newly born cells generated contingently with the late phase. Consequently cells generated during the early phase of learning did not further divide after their labeling with BrdU. The surviving cells differentiated into neurons and the number of 8- to 12-d-old neurons positively correlated with behavioral performances: rats with the highest de novo neuron number were less able to acquire and use spatial information than those with low numbers of new-born neurons. Our present results highlight the importance of this phenomenon in enabling hippocampal learning, given that this step of neurogenesis is altered in aged rats with spatial memory deficits. In young rats, we have shown that the late phase of learning, when asymptotic levels of performances are reached, decreases the survival of cells that are produced during the early phase of learning (Döbrössy et al., 2003). This effect of learning opposed to the learning-promoting effect of learning described above explains why no effects of spatial learning were found when animals are injected with BrdU during both training phases (van Praag et al., 1999; Döbrössy et al., 2003). The learning-induced decrease in cell survival is also observed for a natural form of associative learning, social transmission of food preference (Olariu et al., 2005).

Together, our results and the literature support the hypothesis of a critical period for the learning-induced survival. Newly born cells generated at least 1 week before training might be affected by the learning experience because they are at the adequate period of sensitivity to be rescued by learning (Greenough et al., 1999; Pham et al., 2005). In contrast, cells generated during the early phase of learning might be too young and/or may not have reached adequate maturational stages to be rescued by learning stimuli and, consequently, die. However, whether the surviving newly born neurons participate or not in the memory process remains an open question. The manifestation of learning-related increase in neurogenesis does not prove that they are needed for ongoing behavior. Nevertheless, the observation that the learning-promoting surviving effect is observed specifically in the AU animals strongly suggests that these newly born neurons participate in memory processing. "How" they achieved this goal is an unresolved issue, given that it seems that newborn neurons need several weeks before reaching full functional maturation (Laplagne et al., 2006). This discrepancy may be attributable to the fact that this conclusion is based on retrovirus labeling, which may delay neuronal development. Alternatively, functional maturation of newborn neurons could be accelerated by learning. Finally, the unique properties of adult-generated neurons (they are more excitable than those born during embryogenesis and exhibit unique responses to external stimuli) (Wang et al., 2000; Ambrogini et al., 2004; Schmidt-Hieber et al., 2004) may qualify them to participate in memory processing before achieving full maturation.

In conclusion, our results highlight the importance of learning-induced changes of adult-born cells survival in memory. This bidirectional plasticity is consistent with the "use it or lose it" principle and indicates that new neurons should "learn to survive" (Greenough et al., 1999). Considering hippocampal function, this metaplasticity could be necessary to avoid "catastrophic interference" (Greenough et al., 1999; Wiskott et al., 2006). Finally, our results also provide new insights into the possible neural mechanisms underlying the aging of memory functions by showing that a specific alteration to these developmental steps of adult neurogenesis underlies deficits in spatial memory.

\section{References}

Abrous DN, Koehl M, Le Moal M (2005) Adult neurogenesis: from precursors to network and physiology. Physiol Rev 85:523-569.

Abrous DN, Wojtowicz JM (2007) Neurogenesis and hippocampal memory system. Cold Spring Harbor Laboratory, in press.

Aimone JB, Wiles J, Gage FH (2006) Potential role for adult neurogenesis in the encoding of time in new memories. Nat Neurosci 9:723-727.

Ambrogini P, Orsini L, Mancini C, Ferri P, Ciaroni S, Cuppini R (2004) Learning may reduce neurogenesis in adult rat dentate gyrus. Neurosci Lett 359:13-16.

Döbrössy MD, Drapeau E, Aurousseau C, Le Moal M, Piazza PV, Abrous DN (2003) Differential effects of learning on neurogenesis: learning increases or decreases the number of newly born cells depending on their birth date. Mol Psychiatry 8:974-982.

Drapeau E, Mayo W, Aurousseau C, Le Moal M, Piazza PV, Abrous DN (2003) Spatial memory performances of aged rats in the water maze predict levels of hippocampal neurogenesis. Proc Natl Acad Sci USA 100:14385-14390.

Endl E, Hollmann C, Gerdes J (2001) Antibodies against the Ki-67 protein: 
assessment of the growth fraction and tools for cell cycle analysis. Methods Cell Biol 63:399-418.

Fischer W, Chen KS, Gage FH, Bjorklund A (1992) Progressive decline in spatial learning and integrity of forebrain cholinergic neurons in rats during aging. Neurobiol Aging 13:9-23.

Gage FH, Bjorklund A, Stenevi U, Dunnett SB, Kelly PA (1984) Intrahippocampal septal grafts ameliorate learning impairments in aged rats. Science 225:533-536.

Gage FH, Chen KS, Buzsaki G, Armstrong D (1988) Experimental approaches to age-related cognitive impairments. Neurobiol Aging 9:645-655.

Gallagher M, Burwell R, Burchinal M (1993) Severity of spatial learning impairment in aging: development of a learning index for performance in the Morris water maze. Behav Neurosci 107:618-626.

Geinisman Y, Toledo-Morrell L, Morrell F (1986) Aged rats need a preserved complement of perforated axospinous synapses per hippocampal neuron to maintain good spatial memory. Brain Res 398:266-275.

Gould E, Beylin A, Tanapat P, Reeves A, Shors TJ (1999) Learning enhances adult neurogenesis in the hippocampal formation. Nat Neurosci 2:260-265.

Grady CL, Craik FI (2000) Changes in memory processing with age. Curr Opin Neurobiol 10:224-231.

Greenough WT, Cohen NJ, Juraska JM (1999) New neurons in old brains: learning to survive? Nat Neurosci 2:203-205.

Hairston IS, Little MT, Scanlon MD, Barakat MT, Palmer TD, Sapolsky RM, Heller HC (2005) Sleep restriction suppresses neurogenesis induced by hippocampus-dependent learning. J Neurophysiol 94:4224-4233.

Heine VM, Maslam S, Joels M, Lucassen PJ (2004) Prominent decline of newborn cell proliferation, differentiation, and apoptosis in the aging dentate gyrus, in absence of an age-related hypothalamus-pituitaryadrenal axis activation. Neurobiol Aging 25:361-375.

Kempermann G, Kuhn HG, Gage FH (1997) More hippocampal neurons in adult mice living in an enriched environment. Nature 386:493-495.

Kempermann G, Kuhn HG, Gage FH (1998) Experience-induced neurogenesis in the senescent dentate gyrus. J Neurosci 18:3206-3212.

Kempermann G, Gast D, Gage FH (2002) Neuroplasticity in old age: sustained fivefold induction of hippocampal neurogenesis by long-term environmental enrichment. Ann Neurol 52:135-143.

Laplagne DA, Esposito MS, Piatti VC, Morgenstern NA, Zhao C, van Praag H, Gage FH, Schinder AF (2006) Functional convergence of neurons generated in the developing and adult hippocampus. PLoS Biol 4:e409.

Lemaire V, Aurousseau C, Le Moal M, Abrous DN (1999) Behavioural trait of reactivity to novelty is related to hippocampal neurogenesis. Eur J Neurosci 11:4006-4014.

Lemaire V, Koehl M, Le Moal M, Abrous DN (2000) Prenatal stress produces learning deficits associated with an inhibition of neurogenesis in the hippocampus. Proc Natl Acad Sci USA 97:11032-11037.

Lichtenwalner RJ, Forbes ME, Bennett SA, Lynch CD, Sonntag WE, Riddle DR (2001) Intracerebroventricular infusion of insulin-like growth
factor-I ameliorates the age-related decline in hippocampal neurogenesis. Neuroscience 107:603-613.

Markowska AL, Stone WS, Ingram DK, Reynolds J, Gold PE, Conti LH, Pontecorvo MJ, Wenk GL, Olton DS (1989) Individual differences in aging: behavioral and neurobiological correlates. Neurobiol Aging 10:31-43.

Montaron MF, Drapeau E, Dupret D, Kitchener P, Aurousseau C, Le Moal M, Piazza PV, Abrous DN (2006) Lifelong corticosterone level determines age-related decline in neurogenesis and memory. Neurobiol Aging 27:645-654.

Nacher J, Alonso-Llosa G, Rosell DR, McEwen BS (2003) NMDA receptor antagonist treatment increases the production of new neurons in the aged rat hippocampus. Neurobiol Aging 24:273-284.

Olariu A, Cleaver KM, Shore LE, Brewer MD, Cameron HA (2005) A natural form of learning can increase and decrease the survival of new neurons in the dentate gyrus. Hippocampus 15:750-762.

Pham K, McEwen BS, Ledoux JE, Nader K (2005) Fear learning transiently impairs hippocampal cell proliferation. Neuroscience 130:17-24.

Piazza PV, Deminiere JM, Le Moal M, Simon H (1989) Factors that predict individual vulnerability to amphetamine self-administration. Science 245:1511-1513.

Rapp PR, Amaral DG (1992) Individual differences in the cognitive and neurobiological consequences of normal aging. Trends Neurosci 15:340-345.

Redish AD, Touretzky DS (1998) The role of the hippocampus in solving the Morris water maze. Neural Comput 10:73-111.

Schmidt-Hieber C, Jonas P, Bischofberger J (2004) Enhanced synaptic plasticity in newly generated granule cells of the adult hippocampus. Nature 429:184-187.

Shors TJ, Miesegaes G, Beylin A, Zhao M, Rydel T, Gould E (2001) Neurogenesis in the adult is involved in the formation of trace memories. Nature 410:372-376

Small SA (2001) Age-related memory decline: current concepts and future directions. Arch Neurol 58:360-364.

Smith TD, Adams MM, Gallagher M, Morrison JH, Rapp PR (2000) Circuit-specific alterations in hippocampal synaptophysin immunoreactivity predict spatial learning impairment in aged rats. J Neurosci 20:6587-6593.

Stoelzel CR, Stavnezer AJ, Denenberg VH, Ward M, Markus EJ (2002) The effects of aging and dorsal hippocampal lesions: performance on spatial and nonspatial comparable versions of the water maze. Neurobiol Learn Mem 78:217-233.

van Praag H, Kempermann G, Gage FH (1999) Running increases cell proliferation and neurogenesis in the adult mouse dentate gyrus. Nat Neurosci 2:266-270.

Wang S, Scott BW, Wojtowicz JM (2000) Heterogenous properties of dentate granule neurons in the adult rat. J Neurobiol 42:248-257.

Wiskott L, Rasch MJ, Kempermann G (2006) A functional hypothesis for adult hippocampal neurogenesis: avoidance of catastrophic interference in the dentate gyrus. Hippocampus 16:329-343. 